Supporting Information

\title{
Bioinspired Free-Standing One Dimensional Photonic Crystals with Janus Wettability for Water Quality Monitoring
}

Cihui Liu, Lulu Zhang, Xinran Zhang, Yizhen Jia, Yunsong Di* and Zhixing Gan*

School of Physics and Technology, Nanjing Normal University, Nanjing 210023,

People's Republic of China

*Corresponding author

E-mail address: diyunsong@njnu.edu.cn and zxgan@njnu.edu.cn

\section{Bragg-Snell Formula:}

$m \lambda_{\text {Bragg }}=2 D\left(n^{2} \text { eff }^{-} \sin ^{2} \theta\right)^{1 / 2}$

$n^{2}{ }_{\text {eff }}=n_{1}^{2} f_{1}+n_{2}^{2} f_{2}$

Where $m$ is the diffraction order, $\mathrm{D}$ is the period, $\lambda_{\mathrm{Bragg}}$ is the position of Bragg peak, $\theta$ is the incident angle, $n_{\text {eff }}$ is the effective refractive index, and $n_{1}, f_{1}, n_{2}$ and $f_{2}$ are refractive indexes and volume fractions of the two different layers respectively.

\section{Theoretical value of the stop bands}

According to the formula (1) and (2) above, we can calculate the theoretical value of the photonic stop bands. The effective refractive index of the $\mathrm{TiO}_{2}$ layer was determined by spectroscopic ellipsometry to be 1.831 . The effective refractive index 
of the P(NiPAAm-bis-AA) layer was determined by spectroscopic ellipsometry to be 1.6. The average thicknesses of polymer layer and $\mathrm{TiO}_{2}$ layer are $160 \mathrm{~nm}$ and $70 \mathrm{~nm}$ respectively which were observed by SEM.

\section{Movie S-1}

This movie shows that during the rod stirring process, the Janus 1DPCs film was floating stably on the air-water interface, and the water wave cannot swamp the film. The Janus film successfully floated on and tightly adhered to the water surface using the superhydrophobic/superhydrophilic Janus strategy. It is reasonable that the lower superhydrophilic surface of Janus film was well wetted by water without an air-channel, and therefore film tightly adhered to the water surface. The current finding on this antiroll ability should offer a new opportunity for practical application. 